\title{
Review of: "Polyunsaturated fatty acid arachidonic acid increases the virulence of gut pathogen, Yersinia enterocolitica"
}

\author{
Yolande T R Proroga ${ }^{1}$ \\ 1 Istituto Zooprofilattico Sperimentale del Mezzogiorno
}

Potential competing interests: The author(s) declared that no potential competing interests exist.

PUFA plays an important role in the human diet, the Authors, in this paper

highlighted the role of Arachidonic acid in the alteration of the pathogenesis of Y. enterocolitica. The Introduction as the paragraph material and method was clear and detailed. Results and discussion were exhaustive. Despite, I suggest to review the enumeration of figures? 